\title{
MEDIDAS DEL ANCHO DE LA TABLA ÓSEA VESTIBULAR Y LINGUAL DE LA ZONA ANTEROINFERIOR DE LA MANDÍBULA CON TOMOGRAFÍA CONE BEAM EN PACIENTES ADULTOS
}

\author{
MEASUREMENTS THE WIDTH OF THE BUCCAL AND LINGUAL \\ BONE PLATE OF THE LOWER ANTERIOR AREA OF THE MANDIBLE \\ WITH CONE BEAM TOMOGRAPHY IN ADULT PATIENTS
}

\author{
JHONATAN CANO MANYARI \\ Universidad Norbert Wiener \\ OMAR ALARCÓN PALACIOS \\ Universidad Norbert Wiener \\ GINA LEÓN UNTIVEROS \\ Universidad Norbert Wiener
}

\section{RESUMEN}

El camuflaje en ortodoncia es una alternativa no quirúrgica para solucionar maloclusiones. En estos casos, los dientes donde se ejerce mayor biomecánica son los anteroinferiores, por lo que es importante tener en cuenta la anatomía ósea en esta zona. Por tanto, nuestro objetivo es hallar medidas de la tabla ósea lingual y vestibular de la zona anteroinferior de la mandíbula con ayuda de la tomografía computarizada Cone Beam (TCCB). La muestra estuvo compuesta por un total de 30 pacientes a los que se les realizó una TCCB como parte del diagnóstico ortodóntico. El tomógrafo utilizado fue Sirona y las imágenes fueron procesadas mediante el software PointNixt RealScan 2.0-CDViewer.

Las medidas muestran un aumento de la tabla vestibular en la maloclusión clase III, mientras que en la maloclusión clase II aumenta la tabla lingual; en cuanto a las mediciones de la reproducibilidad interexaminador, estas resultaron elevadas siendo los coeficientes de correlación interclase [ICC $\geq 0.99$ ].

Debemos tener presente la anatomía ósea de los maxilares cuando vamos a camuflar con ortodoncia una maloclusión. La TCCB nos permite realizar mediciones de manera fiable, por lo que pueden ser empleados como registro de diagnóstico en el ámbito de la ortodoncia.

Palabras clave: tomografía computarizada Cone Beam, medición, tabla ósea.

\section{ABSTRACT}

The camouflage orthodontics is a non-surgical alternative to solve bad occlusions, in these cases, teeth where it worked greater biomechanics are the lower front so it is important to take into account the anatomy bone in this area. We will find measures of the lingual bone table and vestibular of the lower anterior region of the mandible with the aid of the Cone Beam computed tomography (CBCT). The sample was 
composed by a total of 30 patients who underwent a CBCT as part of its diagnostic orthodontic. The scanner used was Sirona and images were processed using the software PointNixt RealScan 2.0-CDViewer.

Measures show an increase of vestibular table in class III malocclusion, while in malocclusion class II increases the lingual table, in terms of measurement reproducibility inter examiner was elevated to being the [ICC $\geq 0.99]$.

We must have to present the anatomy bone of the jaws when we go to camouflage with orthodontics to a malocclusion. The CBCT will allows make measurements of way reliable by what may be used as registration diagnostics in the field of the orthodontics.

Key words: Cone Beam Tomography Computed, bone table.

\section{INTRODUCCIÓN}

La parte más importante del tratamiento ortodóntico es el diagnóstico, y en él, una de las ayudas imagenológicas es la TCCB. Se ha comprobado que nos proporciona una buena calidad de imagen y mayor resolución, ya que nos permite hacer cortes más $\operatorname{cortos}^{1,2,3}$.

En ortodoncia y ortopedia maxilar se está haciendo casi indispensable el uso de la TCCB para tener un diagnóstico preciso, pues ayuda en la evaluación de la articulación temporomandibular (ATM), ubicación de dientes retenidos, evaluación de la tabla ósea, asimetría facial ${ }^{4,5}$.

Realizar mediciones pequeñas, como por ejemplo cambios en el espesor del hueso bucal, debe tener algunas consideraciones y entrenamiento, ya que puede haber factores que nos lleven a errores, tales como imágenes fantasmas. Los ajustes utilizados en la TCCB y un análisis de poder estadístico deben ser reportados en los estudios que investigan los pequeños cambios óseos. Por tanto, los protocolos deben establecerse y seguirse para reducir al mínimo la mala interpretación de los resultados y mejorar la calidad de la investigación ${ }^{6}$. Los valores dimensionales encontrados en este estudio marcan una disminución en la altura de la cortical vestibular y disminución del grosor del hueso esponjoso vestibular y lingual, como características principales en pacientes clase III esquelética; así, deben tomarse las debidas precauciones al realizarse los movimientos ortodóncicos para no ocasionar una mayor disminución de la cresta alveolar, y evitar también provocar alguna dehiscencia o fenestración, como señalaron Vierna et al. ${ }^{7}$

Asimismo, Garlock et al. encontraron que el tratamiento de ortodoncia causa cambios en la altura del hueso alveolar y el espesor del hueso cortical, alrededor de los incisivos inferiores. El tratamiento de ortodoncia causa cambios en la altura del hueso alveolar y en el espesor óseo cortical alrededor del incisivo mandibular, aunque en el pretratamiento la inclinación de los incisivos no se correlacionó con los cambio en la altura del hueso alveolar ${ }^{8}$.

\section{MATERIALES Y MÉTODO}

Para el estudio fueron seleccionados 30 pacientes del centro radiológico Teramax, a los cuales se le realizaron TCCB como parte de su diagnóstico ortodóntico; de ellos, 10 registraron maloclusión clase I de angle, 
10 con clase II y 10 con clase III. Las edades comprendían entre los 18 y 40 años.

Los criterios de inclusión en el presente estudio fueron los siguientes:

- Pacientes que no hubieran recibido tratamiento ortodóntico previamente.

- Ausencia de anomalías en el número y forma dentaria.

- Ausencia de enfermedades oseodegenerativas.

- Presencia de los dientes anteroinferiores.

Las tomografías computarizadas Cone Beam de todos los pacientes fueron obtenidas con el tomógrafo Sirona ${ }^{\oplus}$. Este tomógrafo cuenta con dos modalidades, según su campo de visión (FOV): estándar y amplia.

Para la realización del presente estudio se empleó la dimensión amplia para la cabeza completa 200 x 190 mm (14 bits). El tiempo de escaneado fue de 20 segundos, puesto que el modo empleado fue de calidad alta. El ángulo de escaneado cubre los $360^{\circ}$ y genera un número de imágenes de cortes que para el FOV amplio fue de 592. El tamańo de los vóxeles es de $0,5 \mathrm{~mm}$, con un voltaje del tubo de $60 \mathrm{Kv}$ y una intensidad de $6 \mathrm{~mA}$ Posteriormente, los datos sin procesar y los cortes obtenidos de los TCCB de los pacientes fueron introducidos en el software PointNixt RealScan 2.0-CDViewer ${ }^{\bullet}$. Este programa se utiliza para realizar los cortes y obtener imágenes en 3D (Figura 1).

\section{FIGURA 1}

Software PointNixt RealScan 2.0-CDViewer ${ }^{\circledast}$

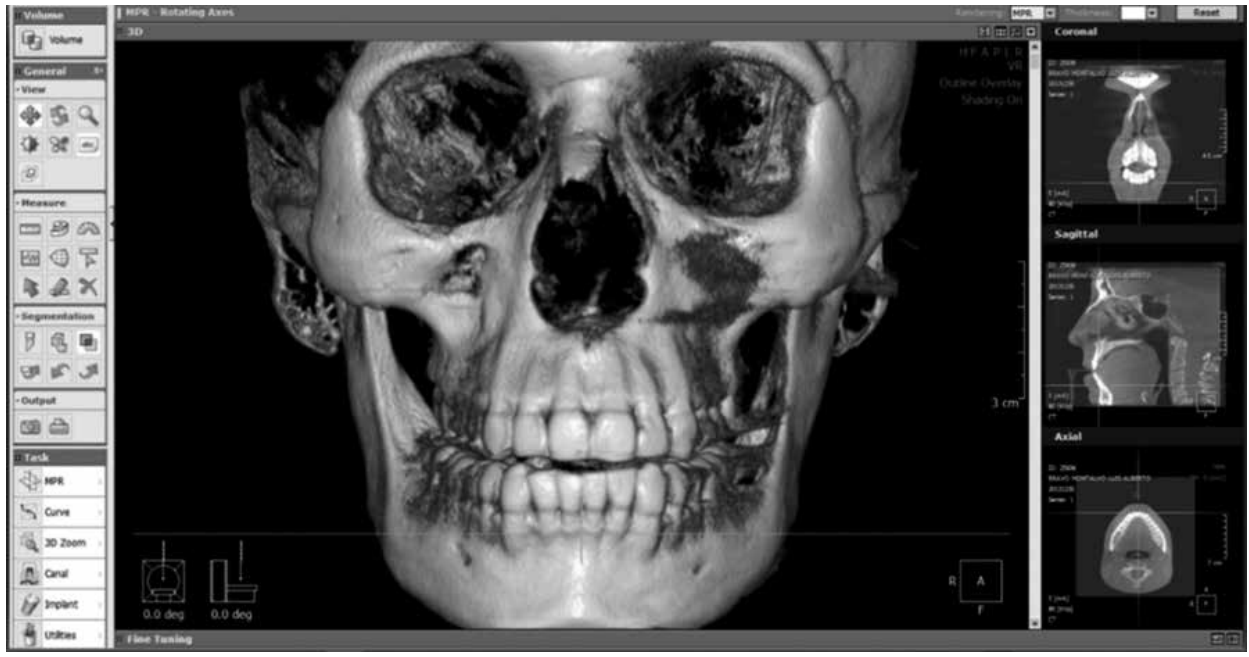


Realizamos los cortes tomográficos a la altura del centro de resistencia de los dientes anteroinferiores, como se puede apreciar en la Figura 2.
Luego ubicamos la imagen en un corte axial y realizamos las siguientes medidas para los dientes 43, 42. 41, 31, 32, 33:

\section{FIGURA 2}

Ubicación aproximada del centro de resistencia de los dientes anteroinferioes

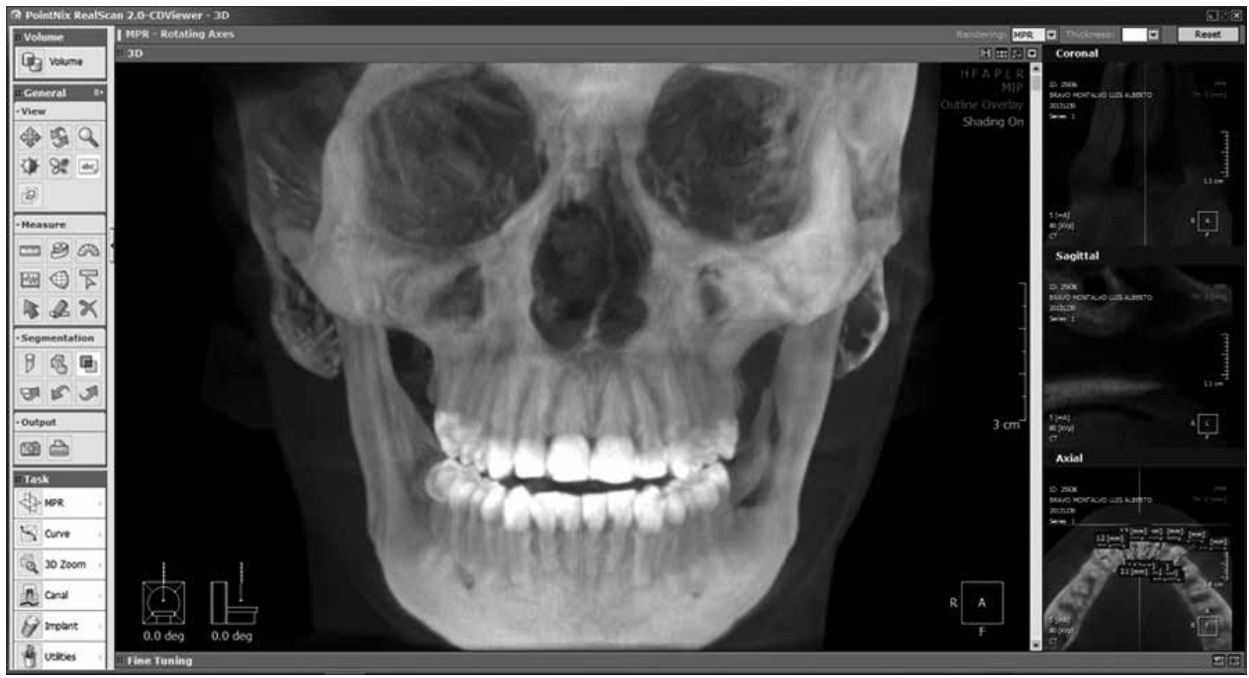

FIGURA 3

Medidas realizadas a las piezas 43, 42, 41, 31, 32, 33.

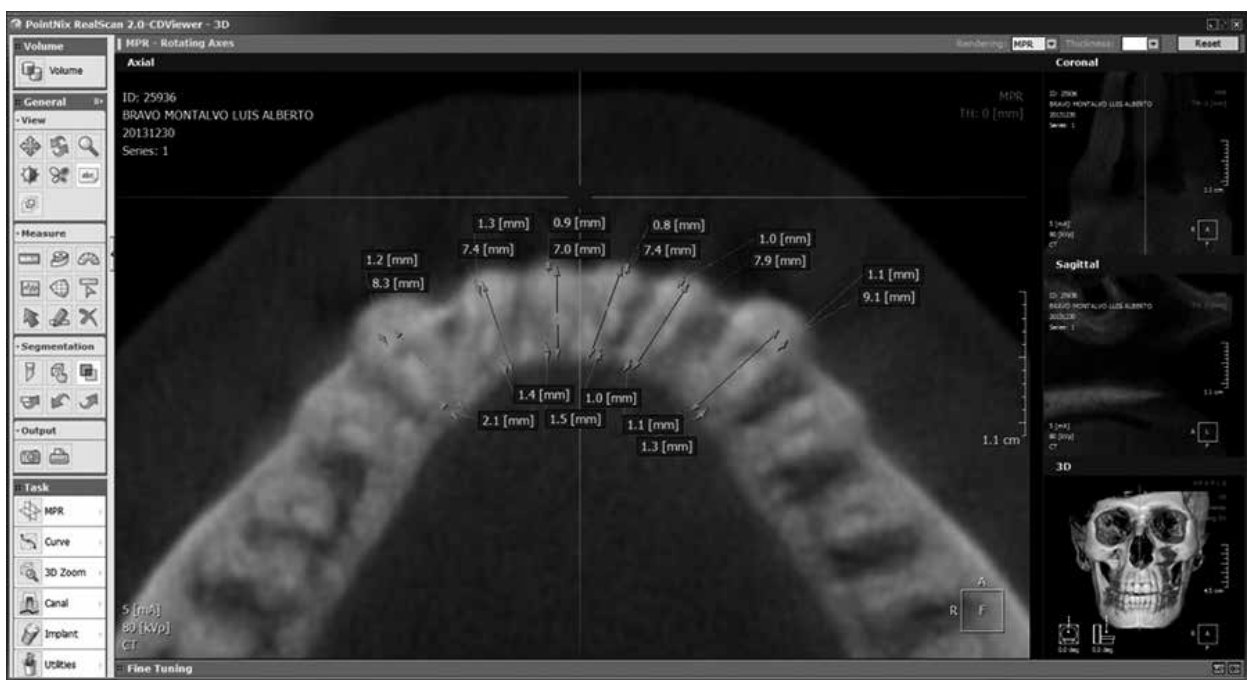


- Desde la cara vestibular radicular hasta el borde más anterior del hueso alveolar mandibular.

- Desde la cara lingual radicular hasta el borde más posterior del hueso alveolar mandibular.

- Desde el borde más anterior hasta el más posterior del hueso alveolar. (Figura 3)

\section{ANÁLISIS ESTADÍSTICO}

Para el proceso de calibración se calcularon también el coeficiente de correlación interclase $(\mathrm{CCI})$, [CCI=0.99].

El presente trabajo se realizó en la base de datos Excel 2010 y se utilizó el programa estadístico SPSS v.21. Se hallaron medidas de tendencia central y dispersión, tales como desviación estándar, media, valor máximo y mínimo e intervalo de confianza.

\section{DISCUSIÓN}

En la actualidad, prácticamente todos los equipos de TCCB que se comercializan son capaces de proporcionar imágenes 1:1 $y$, por tanto, capaces de localizar con exactitud puntos anatómicos, puntos cefalométricos y puntos dentales. Vistos clínicamente, son sistemas adecuados tanto para la localización de dichos puntos como para la realización de medidas lineales, ángulos, diámetros, etcétera ${ }^{9,10}$.

Actualmente, con la incorporación del TCCB a la clínica ortodóntica, existe una nueva alternativa para realizar estas mediciones; sin embargo, en el presente estudio hemos considerado la calibración como un elemento importante para poder reproducir el mismo punto seleccionado en todas las medidas que realicemos, con el fin de contar con un buen entrenamiento para tener la menor variación posible inter- o intraoperador ${ }^{11}$.

En cuanto a las características anatómicas del alveolo, se ha demostrado que una sínfisis delgada está asociada con hueso cortical más delgado ${ }^{12,13}$, y cuando el grosor del hueso cortical disminuye, también lo hace la densidad ósea ${ }^{14}$. Por tanto, en pacientes con crestas más delgadas y hueso cortical menos denso, el alveolo podría ser más propenso a las microfracturas asociadas con el movimiento del diente, dando como resultado un aumento de la pérdida ósea vertical ${ }^{15}$. Kohakura et al. evaluaron la cortical del hueso en sujetos vivos, comparando tres grupos con diferentes dimensiones verticales faciales y utilizando TCCB: mostraron que cuanto más pequeño el gonial y ángulos planos mandibulares, más gruesa es la cortical bucal. También se demostró que esto se aplicaba al hueso cortical bucal superior, particularmente alrededor del premolar y las regiones caninas ${ }^{16}$.

Asimismo, Tesunori et al, en un estudio de 39 varones asiáticos encontraron que el hueso cortical del grupo de cara corta era más grueso que el promedio, y en la superficie lingual del primer y segundo molares; también se encontró que la base de la mandíbula, en los incisivos, tenía un hueso cortical de mayor grosor en el grupo de cara corta en contraste con el grupo de cara lar$\mathrm{ga}^{17}$. Masumoto et al. estudiaron la región molar de 31 cráneos japoneses masculinos, y encontraron que el grupo de cara corta tenía placas corticales más gruesas que los grupos de la cara media o larga en el vestibular, lingual y base de la mandíbula ${ }^{18,19}$.

Dichos estudios encontraron resultados similares a los de la presente investi- 


\section{TABLA 1}

Valores tomados para la maloclusión clase I

\begin{tabular}{|c|c|c|c|c|c|c|c|c|c|c|c|c|c|c|c|}
\hline$N^{\circ}$ Pieza & & Media & & & DS & & & Vmax & & & Vmin & & & IC \% & \\
\hline & lit & lv & ll & lit & lv & 11 & lit & lv & 11 & lit & lv & $\mathrm{Ll}$ & lit & lv & $\mathrm{Ll}$ \\
\hline 43 & 7.05 & 1.87 & 1.95 & 0.20 & 0.15 & 0.15 & 7.4 & 2.1 & 2.2 & 6.8 & 1.6 & 1.7 & 0.12 & 0.09 & 0.09 \\
\hline 42 & 6.77 & 1.59 & 1.61 & 0.18 & 0.11 & 0.09 & 7.2 & 1.8 & 1.8 & 6.6 & 1.4 & 1.5 & 0.11 & 0.06 & 0.06 \\
\hline 41 & 6.69 & 1.57 & 1.55 & 0.17 & 0.12 & 0.12 & 7 & 1.7 & 1.8 & 6.5 & 1.3 & 1.4 & 0.10 & 0.07 & 0.07 \\
\hline 31 & 6.98 & 1.54 & 1.55 & 0.16 & 0.16 & 0.11 & 7 & 1.8 & 1.7 & 6.4 & 1.3 & 1.4 & 0.10 & 0.10 & 0.07 \\
\hline 32 & 6.7 & 1.54 & 1.61 & 0.22 & 0.15 & 0.12 & 7.1 & 1.8 & 1.8 & 6.4 & 1.3 & 1.4 & 0.14 & 0.09 & 0.07 \\
\hline 33 & 7.15 & 1.9 & 1.98 & 0.21 & 0.11 & 0.11 & 7.4 & 2.1 & 2.1 & 6.8 & 1.8 & 1.7 & 0.13 & 0.07 & 0.07 \\
\hline
\end{tabular}

Lit: Longitud inter tabla ósea. Lv: Longitud vestibular ósea. Ll: Longitud lingual ósea.

\section{TABLA 2}

Valores tomados para la maloclusión clase II

\begin{tabular}{|c|c|c|c|c|c|c|c|c|c|c|c|c|c|c|c|}
\hline \multirow{2}{*}{$\begin{array}{c}N^{\circ} \text { Pieza } \\
\text { dental }\end{array}$} & \multicolumn{3}{|c|}{ Media } & \multicolumn{3}{|c|}{ DS } & \multicolumn{3}{|c|}{$V \max$} & \multicolumn{3}{|c|}{ Vmin } & \multicolumn{3}{|c|}{ IC \% } \\
\hline & lit & lv & 11 & lit & lv & ll & lit & $\mathrm{lv}$ & ll & lit & lv & $\mathrm{Ll}$ & lit & lv & $\mathrm{Ll}$ \\
\hline 43 & 6.85 & 1.67 & 2.15 & 0.20 & 0.15 & 0.15 & 7.2 & 1.9 & 2.4 & 6.6 & 1.4 & 1.9 & 0.12 & 0.09 & 0.09 \\
\hline 42 & 6.57 & 1.39 & 1.81 & 0.18 & 0.11 & 0.99 & 7 & 1.6 & 1.9 & 6.4 & 1.2 & 1.7 & 0.11 & 0.06 & 0.06 \\
\hline 41 & 6.49 & 1.42 & 1.75 & 0.17 & 0.13 & 0.12 & 6.8 & 1.7 & 1.9 & 6.3 & 1.2 & 1.6 & 0.10 & 0.86 & 0.07 \\
\hline 31 & 6.48 & 1.34 & 1.75 & 0.16 & 0.16 & 0.11 & 6.9 & 1.6 & 1.9 & 6.2 & 1.1 & 1.6 & 0.10 & 0.10 & 0.07 \\
\hline 32 & 6.5 & 1.34 & 1.79 & 0.22 & 0.15 & 0.14 & 6.9 & 1.6 & 1.9 & 6.2 & 1.1 & 1.6 & 0.14 & 0.09 & 0.08 \\
\hline 33 & 6.95 & 1.77 & 2.09 & 0.21 & 0.11 & 0.11 & 7.2 & 1.9 & 2.3 & 6.6 & 1.6 & 1.9 & 0.13 & 0.07 & 0.07 \\
\hline
\end{tabular}

gación, aunque no realizaron la medición especifica realizada en el presente estudio.

\section{CONCLUSIONES}

En base a los resultados obtenidos en nuestro estudio, podemos establecer las siguientes conclusiones:
- La reproducibilidad en el momento de localizar los puntos donde se midieron las distancias de tabla ósea vestibular y lingual hacia los dientes es elevada, con una escala_de error $[\mathrm{CCI}=0,99]$.

- En base a lo investigado, la tabla ósea vestibular es mayor en maloclusiones clase III, en comparación con las de clase I y II. 


\section{TABLA 3}

Valores tomados para la maloclusión clase III

\begin{tabular}{|c|c|c|c|c|c|c|c|c|c|c|c|c|c|c|c|}
\hline$N^{\circ}$ Pieza & & Media & & & DS & & & Vma & & & Vmin & & & IC \% & \\
\hline & lit & lv & 11 & lit & lv & 11 & lit & lv & ll & lit & lv & $\mathrm{Ll}$ & lit & lv & $\mathrm{Ll}$ \\
\hline 43 & 7.04 & 2.07 & 1.75 & 0.15 & 0.15 & 0.15 & 7.3 & 2.3 & 2 & 6.8 & 1.8 & 1.5 & 0.09 & 0.09 & 0.09 \\
\hline 42 & 6.84 & 1.75 & 1.41 & 0.15 & 0.09 & 0.09 & 7.2 & 19 & 1.6 & 6.7 & 1.6 & 1.3 & 0.09 & 0.06 & 0.06 \\
\hline 41 & 6.71 & 1.78 & 1.35 & 0.14 & 0.12 & 0.12 & 7 & 1.9 & 1.6 & 6.5 & 1.5 & 1.2 & 0.08 & 0.07 & 0.07 \\
\hline 31 & 6.68 & 1.74 & 1.38 & 0.16 & 0.15 & 0.15 & 7 & 1.8 & 1.5 & 6.5 & 1.5 & 1.2 & 0.10 & 0.10 & 0.10 \\
\hline 32 & 6.74 & 1.74 & 1.43 & 0.20 & 0.13 & 0.13 & 7.1 & 1.9 & 1.6 & 6.4 & 1.5 & 1.2 & 0.12 & 0.08 & 0.07 \\
\hline 33 & 7.04 & 2.14 & 1.69 & 0.13 & 0.12 & 0.12 & 7.3 & 2.3 & 1.9 & 6.9 & 2 & 1.5 & 0.08 & 0.07 & 0.07 \\
\hline
\end{tabular}

- La longitud ósea intertablas es menor en maloclusiones clase II, en comparación con las de clase I y III.

\section{REFERENCIAS BIBLIOGRÁFICAS}

1. Mozzo P, Procacci C, Tacconi A, Martini PT, Andreis IA. A new volumetric CT machine for dental imaging based on the cone-beam technique: preliminary results. EurRadiol. 1998; 8(9):1558-64.

2. Farman AG, Scarfe WC. Development of imaging selection criteria and procedures should precede cephalometric assessment with cone-beam computed tomography. Am J OrthodDentofacial Orthop. 2006; 130(2):257-65.

3. Mischkowski RA, Pulsfort R, Ritter L, Neugebauer J, Brochhagen H.G, Keeve E, Zöller J.E. Geometric accuracy of a newly developed cone-beam device for maxillofacial imaging. Oral Surg Oral Med Oral Pathol Oral RadiolEndod 2007; 104(4):551-9.

4. Fuentes R, Navarro P, Salamanca C, Cantín M, Garay I, Flores T. Caracterización morfométrica del reborde anterior de la maxila mediante Tomografía computarizada Cone-Beam. Int. J. Morphol., 32(2):493-498, 2014.

5. Nur RB, Germeç Çakan D, Arun T. Evaluation of facial hard and soft tissue asymmetry using cone-beam computed tomography, American Journal of Orthodontics and Dentofacial Orthopedics, Vol. 149, Issue 2, p225-237, 2016.

6. Molen $\mathrm{AD}$. Considerations in the use of cone-beam computed tomography for buccal bone measurements, American Journal of Orthodontics and Dentofacial Orthopedics, Vol. 137, Issue 4, S130S135 Published in issue: April 2010.

7. Vierna J, Cisneros G, Andrade A, Carras R, Vaillard E. Medición del espesor del hueso esponjoso y altura de la cresta alveolar en zona de incisivos inferiores con maloclusión clase III esquelética mediante el uso de tomografía axial computarizada. Rev Tame. 2014; 2 (6):180-183.

8. Garlock DT, Buschang PH, Araujo EA, Behrents RG, Kim KB. Evaluation of marginal alveolar bone in the anterior 
mandible with pretreatment and posttreatment computed tomography in nonextraction patients American Journal of Orthodontics and Dentofacial Orthopedics, Vol. 149, Issue 2, p192201, February 2016.

9. Lou L, Lagravère MO, Compton S, Major PW, Flores-Mir C. Accuracy of measurements and reliability of landmark identification with computed tomography (CT) techniques in the maxillofacial area: a systematic review. Oral Surg Oral Med Oral Pathol Oral RadiolEndod. 2007; 104(3):402-11.

10. Zamora N, Llamas JM, Cibrián R, Gandia JL, Paredes V. A study on the reproducibility of cephalometric landmarks when undertaking a three-dimensional (3D) cephalometric analysis.Med Oral Patol Oral Cir Bucal. 2012;17(4):678-88.

11. Tarazona B, Llamas JM, Cibrian R, Gandia JL, Paredes V. A comparison between dental measurements taken from CBCT models and those taken from a Digital Method.Eur J Orthod. 2013;35(1):1-6.

12. Fuhrmann R. Three-dimensional interpretation of periodontal lesions and remodeling during orthodontic treatment. Part III. J Orofac Orthop 1996;57:224-37.

13. Swasty D, Lee J, Huang JC, Maki K, Gansky SA, Hatcher D, et al. Cross-sectional human mandibular morphology as as- sessed in vivo by cone-beam computed tomography in patients with different vertical facial dimensions. Am J Orthod Dentofacial Orthop 2011;139(Suppl):e377-89.

14. Bresin A, Kiliaridis S, Strid KG. Effect of masticatory function on the internal bone structure in the mandible of the growing rat. Eur J Oral Sci 1999; 107:35-44.

15. Taylor D, Lee TC. Microdamage and mechanical behaviour: predicting failure and remodelling in compact bone. J Anat 2003;203:203-11.

16. Kohakura S, Kasai K, Ohno I, Kanazawa E. Relationship between maxillofacial morphology and morphological characteristics of vertical sections of the mandible obtained by CT scanning. J Nihon Univ School Dent 1997;39:71-7.

17. Tesunori M, Mashita M, Kasai K. Relationship between facial types and tooth and bone characteristics of the mandible obtained by CT scanning. Angle Orthod 1998;68:557-62.

18. Masumoto T, Hayashi I, Kawamura A, Tanaka K, Kasai K. Relationships among facial type, buccolingual molar inclination, and cortical bone thickness of the mandible. Eur J Orthod 2001;23: 15-23.

19. Schubert W, Kobienia BJ, Pollock RA. Cross-sectional area of the mandible. J Oral Maxillofac Surg 1997;55:689-92. 